\title{
Analysis and Design Equations for Phase Matching Using Bragg Reflector Waveguides
}

\author{
Brian R. West and A. S. Helmy, Senior Member, IEEE
}

\begin{abstract}
In this paper, we introduce and analyze a novel waveguide design to provide phase matching for nonlinear optical processes. Phase matching is achieved by designing the structure to guide the fundamental frequency by total internal reflection and the second harmonic (SH) frequency by transverse Bragg reflection. By forcing the SH mode to operate in the middle of the Bragg stopband, we solve for the waveguide dimensions for arbitrary waveguide materials, given the material dispersion between the fundamental and SH frequencies. Using GaAs-AlGaAs as an example, we analytically investigate and quantify properties such as nonlinear coupling efficiency, bandwidth, tunability, and limitations due to dispersion. The technique shows tremendous promise when compared to alternate technologies, where it is particularly attractive as an effective means to obtain ultralow-loss nonlinear optical elements for monolithic integration with coherent light sources and other active devices.
\end{abstract}

Index Terms-Integrated optics, nonlinear optics, optical phase matching, optical waveguides, phase matching.

\section{INTRODUCTION}

$\mathbf{T}$ HE demand on optical devices and technologies in the fields of signal processing, mobile communication technologies, on chip/board data links, aeronautics, aerospace, sensing, life science diagnostics, and environmental monitoring is at an all time high [1]. The availability, performance, and cost effectiveness of such photonic components has been inadequate at best. This can partially be attributed to the lack of an efficient, compact, and tunable nonlinear element in the optics domain in conjunction with a material system with a viable fabrication technology. III-V semiconductors have been widely used in integrated optoelectronic circuits and nonlinear optical applications. Mature growth, lithography, and etching technologies allow the fabrication of low-loss guiding structures. The use of electronic-scale heterostructures enables additional control, flexibility, and functionality to be incorporated into the devices. The large useable nonlinearities achievable to date in III-V semiconductors chiefly rely on carriers, and have been studied extensively to induce nonlinearities in semiconductor optical amplifiers [2]. The drawbacks of high insertion loss, large size, and excessive noise figures are inherently associated with them and hence they will not be suitable for all applications [3]. Most notable applications that would benefit from integrable ultrafast optical nonlinearities include monolithically integrated optical

Manuscript received August 1, 2005. This work was supported by the Natural Sciences and Engineering Research Council of Canada and by the Edward S. Rogers, Sr. Department of Electrical and Computer Engineering at the University of Toronto.

The authors are with the Edward S. Rogers Sr. Department of Electrical and Computer Engineering, University of Toronto, Toronto, ON M5S 3G4, Canada (e-mail: brian.west@utoronto.ca; a.helmy@utoronto.ca).

Digital Object Identifier 10.1109/JSTQE.2006.872733 parametric oscillators (OPOs), correlated photon pair sources, and tunable frequency conversion monolithic arrays.

OPOs have become indispensable coherent sources for the midinfrared. Their operating wavelength span is limited by the transparency window of lithium niobate, however, because periodically poled lithium niobate (PPLN) is the most commonly used nonlinear element in OPOs. In contrast, compound semiconductors such as GaAs exhibit higher nonlinear coefficients near the materials' resonances in comparison to PPLN, and have a large transparency window. In the case of GaAs, the transparency window scans the spectral range of 1-17 $\mu \mathrm{m}$. GaAs also has high damage threshold and a mature fabrication technology for making waveguides as compared to PPLN.

Correlated photon pairs can be generated through the process of parametric down conversion [4]. For example, one photon at $0.775 \mu \mathrm{m}$ injected into a sample with appropriate phase matching could generate two correlated photons at $1.55 \mu \mathrm{m}$ [5]. Achieving this technology provides very compact sources and allows the integration of the pump source on the same chip. This finds applications in fields ranging from metrology, calibration, quantum experiments, to quantum key distribution [6].

Ultrafast nonlinearities can also enable the realization of integrated arrays of tunable frequency conversion elements. Optical telecommunications networks which imply any form of wavelength diversity in the physical layer could greatly benefit from these devices. The tuning offered by these devices, together with the integration of the pump source on the same chip, provides unprecedented versatility and configurability into the network [7]. Devices with such functionality have been previously demonstrated in $\mathrm{LiNbO}_{3}$ and have been proven very successful in wavelength-division multiplexing (WDM) networks [8]. However, monolithically integrated arrays would vastly reduce system complexity, and hence the cost.

It is clear from the few applications discussed that achieving efficient, low-loss, and tunable phase matching in a semiconductor material is pivotal for the realization of the next generation of photonic devices. The high nonlinear coefficients of semiconductors at photon energies near the bandgap are difficult to exploit practically, as the material dispersion is formidable in this spectral region. This makes phase matching between the fundamental and second harmonic ( $\mathrm{SH}$ ) waves difficult to achieve. Various authors have proposed schemes to overcome this limitation, utilizing form birefringence [9], quasi-phase matching (QPM) through periodic suppression of the nonlinear coefficient [10] or domain inversion [11], [12], and resonant cavities [13]. Such methods generally suffer from difficulty in monolithic integration with other active and passive components, or large insertion loss due to scattering. 


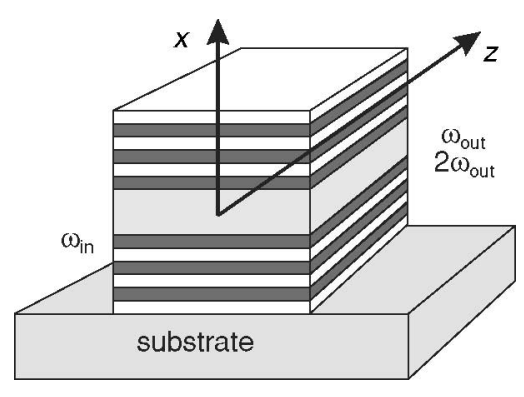

Fig. 1. BRW for phase-matched second harmonic generation.

In this work, we analyze in detail a means which was proposed recently to achieve phase matching through the use of a novel waveguide design [14]. The technique is material independent and requires no patterning in the direction of propagation, which will significantly lower the insertion loss. The waveguide will utilize total internal reflection (TIR) at the fundamental wavelength and transverse Bragg reflection at the $\mathrm{SH}$ wavelength. In Section II, we discuss the Bragg reflection waveguide (BRW) and the solution of its quasi-bound modes, with particular attention to the special case of a transverse quarter-wave cladding. Section III introduces a procedure by which the BRW mode at the SH wavelength can be phase matched with the TIR mode at the fundamental wavelength for arbitrary core and cladding materials. In Section IV, we analyze critical waveguide properties such as dispersion and nonlinear coupling coefficient. Wavelength tuning mechanisms are proposed in Section V, while in Section VI we suggest various modifications to the waveguide design that can improve the device operation.

\section{BRWs}

Although initially proposed earlier, the BRW (Fig. 1) was analyzed by Yariv, Yeh, and Hong [15], [16]. While the more common TIR waveguide operates through total internal reflection between a high-index core and lower index cladding, the BRW has no such requirement; rather, it utilizes the stopband of a transverse distributed Bragg reflector to provide reflection and thus confinement of guided waves, and hence can guide light in a region of any refractive index. The ability to guide light within a low-index core has received significant attention from many authors, particularly in the development of air-core guides for mechanically tunable gratings [17] and particle acceleration [18]. The unique birefringence properties of BRWs due to the polarization dependence of the Fresnel coefficients have been utilized to create polarization splitters/combiners [19]. For nonlinear propagation, BRWs have been shown to support spatial optical solitons [20], as well as nonlinear guided modes at high optical power in structures that support no linear bound modes [21].

In the context of phase matching, the ability of BRWs to support essentially lossless bound modes with effective indices lower than any of the material indices will be utilized for the SH wave to compensate for material dispersion [14]. Strictly speaking, the BRW requires an infinite number of cladding pe-

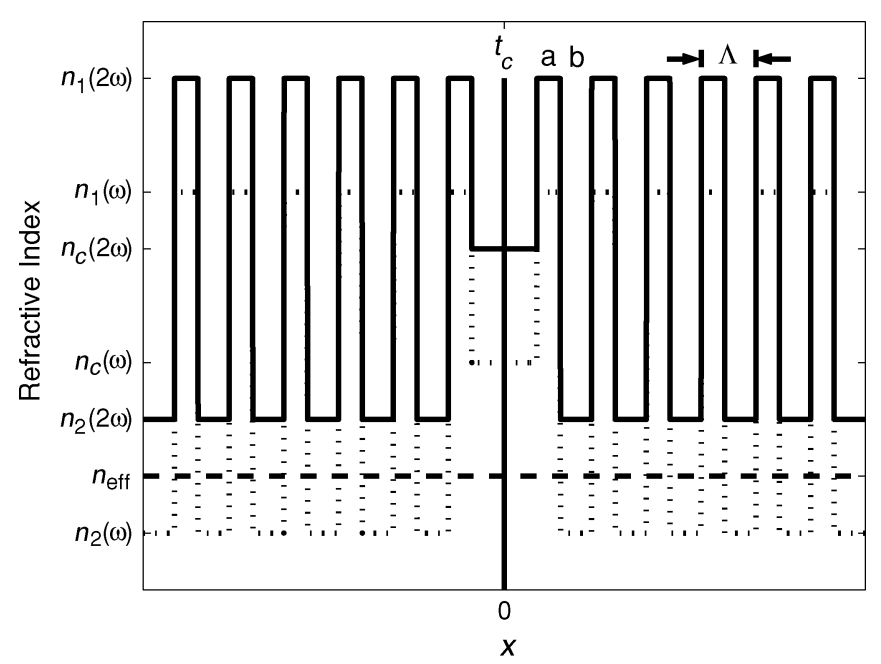

Fig. 2. 1-D BRW with material indices and dimensions indicated. (Solid line) Indices at $2 \omega$. (Dotted line) Indices at $\omega$. The dashed line represents the phasematched effective index.

riods to have zero loss, but as will be shown further, with proper design suitably low loss can be achieved with just a few periods. As such, the derivations in the sections that follow will assume an infinite cladding in order to utilize Bloch-Floquet theory; implications of a finite cladding will be discussed in Section IV.

The one-dimensional (1-D) BRW is shown schematically in Fig. 2. It consists of a core of thickness $t_{\mathrm{c}}$ and index $n_{\mathrm{c}}$, and periodic cladding layers of thickness $a\left(n_{1}\right)$ and $b\left(n_{2}\right)$. The cladding period is $\Lambda=a+b$ and the waveguide is symmetric about $x=0$. BRWs in general need not be symmetric, but this restriction eases the design and ensures strong overlap between the guided modes and the core. Fig. 2 indicates the material indexes at both fundamental and $\mathrm{SH}$ wavelengths as well as the phase-matched effective index.

The effective indices and mode field profiles of the guided modes in this structure are derived explicitly in [22]. Here, we summarize only the pertinent results. For the purpose of phase matching, we will examine only even modes (by parity, odd BRW modes will have zero overlap with the desired even TIR modes, leading to zero second-harmonic generation (SHG) efficiency, as shown in Section IV). In each layer, the transverse wave vector at an angular frequency $\omega$ is defined as

$$
k_{i}=\sqrt{\left(\frac{\omega n_{i}}{c}\right)^{2}-\beta^{2}}
$$

where $i=(1,2, \mathrm{c}), \beta$ is the propagation constant, and $c$ is the velocity of light. The assumption of an infinite cladding implies that $\beta$ is real (absorption in the waveguide is presumed negligible here). Guided modes satisfy the mode dispersion equation

$$
\begin{gathered}
\frac{1}{k_{\mathrm{c}}} \cot \left(\frac{k_{\mathrm{c}} t_{\mathrm{c}}}{2}\right)=\frac{-i}{k_{1}} \frac{e^{i K_{\mathrm{TE}} \Lambda}-A_{\mathrm{TE}}+B_{\mathrm{TE}}}{e^{i K_{\mathrm{TE}} \Lambda}-A_{\mathrm{TE}}-B_{\mathrm{TE}}} \\
\frac{1}{k_{\mathrm{c}}} \cot \left(\frac{k_{\mathrm{c}} t_{\mathrm{c}}}{2}\right)=\frac{-i}{k_{1}}\left(\frac{n_{1}}{n_{\mathrm{c}}}\right)^{2} \frac{e^{i K_{\mathrm{TM}} \Lambda}-A_{\mathrm{TM}}+B_{\mathrm{TM}}}{e^{i K_{\mathrm{TM}} \Lambda}-A_{\mathrm{TM}}-B_{\mathrm{TM}}}
\end{gathered}
$$


In (2), the terms $A$ and $B$ are elements of the unit cell translation matrix of the cladding

$$
\begin{aligned}
A_{\mathrm{TE}} & =e^{i k_{1} a}\left[\cos k_{2} b+\frac{i}{2}\left(\frac{k_{2}}{k_{1}}+\frac{k_{1}}{k_{2}}\right) \sin k_{2} b\right] \\
B_{\mathrm{TE}} & =e^{-i k_{1} a}\left[\frac{i}{2}\left(\frac{k_{2}}{k_{1}}-\frac{k_{1}}{k_{2}}\right) \sin k_{2} b\right] \\
A_{\mathrm{TM}} & =e^{i k_{1} a}\left[\cos k_{2} b+\frac{i}{2}\left(\frac{n_{2}^{2} k_{1}}{n_{1}^{2} k_{2}}+\frac{n_{1}^{2} k_{2}}{n_{2}^{2} k_{1}}\right) \sin k_{2} b\right] \\
B_{\mathrm{TM}} & =e^{-i k_{1} a}\left[\frac{i}{2}\left(\frac{n_{2}^{2} k_{1}}{n_{1}^{2} k_{2}}-\frac{n_{1}^{2} k_{2}}{n_{2}^{2} k_{1}}\right) \sin k_{2} b\right]
\end{aligned}
$$

and $K$ is the Bloch wavenumber of the cladding

$$
K_{\mathrm{TE}(\mathrm{TM})}=\frac{1}{\Lambda} \cos ^{-1}\left[\operatorname{Re}\left(A_{\mathrm{TE}(\mathrm{TM})}\right)\right] .
$$

When $|\operatorname{Re}(A)|>1, K=m \pi / \Lambda+i K_{\mathrm{im}}$, where $m=1,2, \ldots$. indicates the order of Bragg reflection. This implies that the field in the cladding is evanescent, which is a requirement for waveguiding.

\section{A. Quarter-Wave Bragg Reflection Waveguides (QtW-BRWs)}

Now, let us examine the case where each layer of the cladding has quarter-wave optical thickness with respect to the transverse wave vectors $k_{i}$, i.e., $k_{1} a=k_{2} b=\pi / 2$. This constraint places the Bloch wave number $K$ in the middle of the Bragg stopband, thus ensuring strong guiding through maximizing the field decay in the claddings. In this case,

$$
\begin{aligned}
A_{\mathrm{TE}} & =-\frac{1}{2}\left(\frac{k_{2}}{k_{1}}+\frac{k_{1}}{k_{2}}\right) \quad B_{\mathrm{TE}}=\frac{1}{2}\left(\frac{k_{2}}{k_{1}}-\frac{k_{1}}{k_{2}}\right) \\
A_{\mathrm{TM}} & =-\frac{1}{2}\left(\frac{n_{2}^{2} k_{1}}{n_{1}^{2} k_{2}}+\frac{n_{1}^{2} k_{2}}{n_{2}^{2} k_{1}}\right) \quad B_{\mathrm{TM}}=\frac{1}{2}\left(\frac{n_{2}^{2} k_{1}}{n_{1}^{2} k_{2}}-\frac{n_{1}^{2} k_{2}}{n_{2}^{2} k_{1}}\right) \\
e^{i K_{\mathrm{TE}} \Lambda} & =-\left(\frac{k_{2}}{k_{1}}\right) \quad e^{i K_{\mathrm{TM}} \Lambda}=-\left(\frac{n_{1}^{2} k_{2}}{n_{2}^{2} k_{1}}\right) .
\end{aligned}
$$

By inserting (5) into (2) we see that modes occur when

$$
\frac{k_{\mathrm{c}} t_{\mathrm{c}}}{2}=\frac{(p+1) \pi}{2}, \quad p=0,2,4, \ldots
$$

where $p$ is the mode order for even modes. In the quarter-wave case, the electric field has a null at the core/cladding boundary [22]. This result is supported by the observation that the transverse field is encountering an external reflection at a perfect quarter-wave stack of infinite periods for which the reflection coefficient $r=-1$. The sum of outgoing and incoming fields therefore equals zero at the interface, a condition referred to in the literature as a "metallic-like" boundary [23]. We will consider only the fundamental even BRW mode in the next of this paper, for which $p=0$. In this case,

$$
k_{\mathrm{c}}=\frac{\pi}{t_{\mathrm{c}}}=\frac{2 \pi}{\lambda} \sqrt{n_{\mathrm{c}}^{2}-n_{\mathrm{eff}}^{2}}
$$

and thus

$$
n_{\mathrm{eff}}=\sqrt{n_{\mathrm{c}}^{2}-\left(\frac{\lambda}{2 t_{\mathrm{c}}}\right)^{2}} .
$$

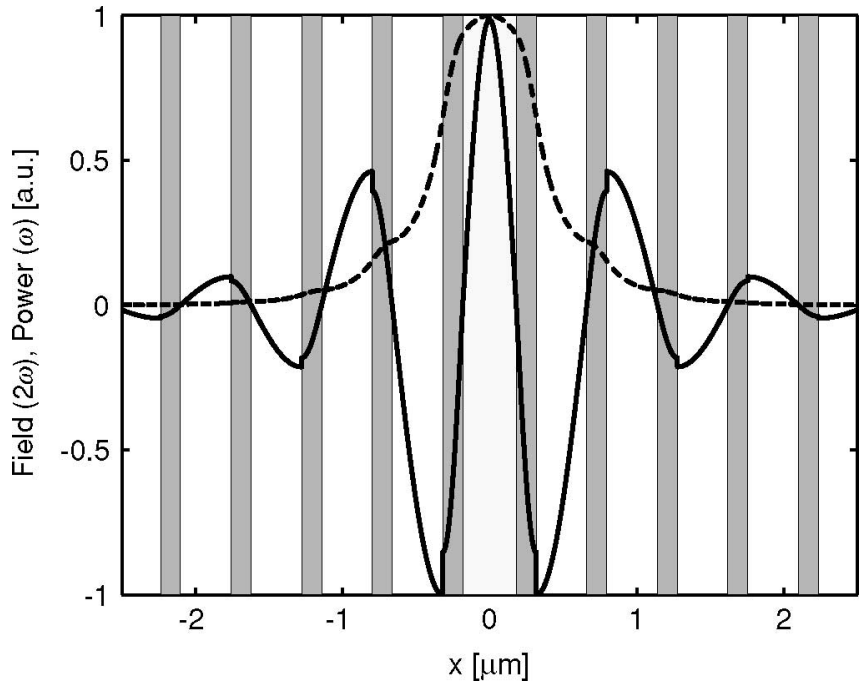

Fig. 3. (Solid line) field profile of SH mode and (dashed line) power profile of phase-matched fundamental TIR mode in a BRW that is quarter-wave at the SH wavelength.

From (8), it can be seen that for a given core index, a waveguide can be designed to have an arbitrary effective index simply by adjusting the core thickness. It must be noted, however, that the effective index cannot exceed the index of the cladding material. If this condition is violated, the field will be evanescent in this material and quarter-wave thickness cannot be achieved [24]. In addition, (8) is only valid when the argument of the square root is positive. For $n_{2}<n_{\mathrm{c}}<n_{1}$, these conditions constrain the guide width to

$$
\frac{\lambda}{2 n_{\mathrm{c}}}<t_{\mathrm{c}}<\frac{\lambda}{2 \sqrt{n_{\mathrm{c}}^{2}-n_{2}^{2}}} .
$$

Fig. 3 shows the field profile of a quarter-wave TM Bragg mode at the SH frequency and the power profile in the TE TIR mode at the fundamental frequency. The justification for this representation will become clear in Section IV-A.

Now that we have an analytical form for calculating the effective index of the BRW mode, we should be able to solve it simultaneously with that of the TIR mode within the same waveguide at one-half of the photon energy. The design procedure for calculating the effective index of the BRW mode with that of the TIR mode is discussed in Section III.

\section{Phase-MATChed Modes}

The previous section provided a mode dispersion equation (2) for the BRW. In this section we establish a self-consistent design procedure to match the effective indices of a QtW-BRW mode at the SH frequency $(2 \omega)$ with a TIR mode at the fundamental frequency $(\omega)$ when the core and cladding materials are known, using a suitable choice of core thickness, as shown in Fig. 4. Note that the cladding thicknesses are implicitly variable - they will be determined from the resulting effective index using the quarter-wave condition. In actuality, the TIR mode is confined by the Bragg reflection in the cladding as well as internal reflection at the cladding interfaces $\left(n_{\text {eff }}>n_{2}^{\omega}\right)$, so the waveguide can 


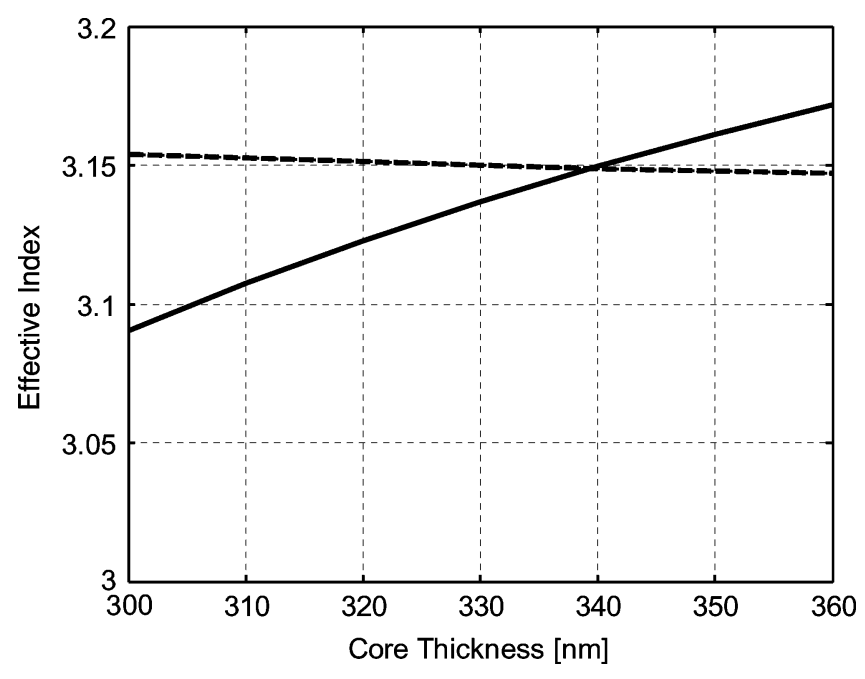

Fig. 4. Effective index versus core thickness for a waveguide with $\left\{x_{1}, x_{2}, x_{\mathrm{c}}\right\}=\{0.3,0.5,0.4\}$. (Solid line) BRW mode, (dashed line) TIR mode. Phase matching occurs where the curves cross. Cladding layer thicknesses are determined by the quarter-wave condition of the BRW mode.

still be modeled using the results of Section II. For this example, we consider type-I phase matching, with TE polarization for the fundamental $(\omega)$ mode and TM polarization for the SHG $(2 \omega)$ mode. The methodology is applicable to type-II phase matching as well, when the appropriate mode dispersion equation (2) is used.

In all the equations given below, the superscripts indicate which frequency ( $\omega$ or $2 \omega$ ) is under consideration. Values that are identical for both frequencies $\left(a, b\right.$, and $\left.n_{\text {eff }}\right)$ are not superscripted. We begin by expressing (8) in terms of frequency

$$
n_{\mathrm{eff}}=\sqrt{\left(n_{\mathrm{c}}^{2 \omega}\right)^{2}-\left(\frac{\pi c}{2 \omega t_{\mathrm{c}}}\right)^{2}} .
$$

Cladding layer thicknesses $a$ and $b$ are determined by the quarter-wave condition at $2 \omega$

$$
\begin{aligned}
& a=\frac{\pi}{2 k_{1}^{2 \omega}}=\frac{\pi c}{4 \omega}\left(\left(n_{1}^{2 \omega}\right)^{2}-n_{\mathrm{eff}}^{2}\right)^{-1 / 2} \\
& b=\frac{\pi}{2 k_{2}^{2 \omega}}=\frac{\pi c}{4 \omega}\left(\left(n_{2}^{2 \omega}\right)^{2}-n_{\mathrm{eff}}^{2}\right)^{-1 / 2}
\end{aligned}
$$

and transverse propagation vectors at $\omega$ are determined as

$$
\begin{aligned}
k_{\mathrm{c}}^{\omega} & =\frac{\omega}{c} \sqrt{\left(n_{\mathrm{c}}^{\omega}\right)^{2}-n_{\mathrm{eff}}^{2}} \\
k_{1}^{\omega} & =\frac{\omega}{c} \sqrt{\left(n_{1}^{\omega}\right)^{2}-n_{\mathrm{eff}}^{2}} \\
k_{2}^{\omega} & =\frac{\omega}{c} \sqrt{\left(n_{2}^{\omega}\right)^{2}-n_{\mathrm{eff}}^{2}}
\end{aligned}
$$

where, for a TIR guide, $k_{2}^{\omega}$ is imaginary. Unit cell translation matrix elements at $\omega$ (for TE polarization) are as in (3)

$$
\begin{aligned}
& A_{\mathrm{TE}}^{\omega}=e^{i k_{1}^{\omega} a}\left[\cos k_{2}^{\omega} b+\frac{i}{2}\left(\frac{k_{2}^{\omega}}{k_{1}^{\omega}}+\frac{k_{1}^{\omega}}{k_{2}^{\omega}}\right) \sin k_{2}^{\omega} b\right] \\
& B_{\mathrm{TE}}^{\omega}=e^{-i k_{1}^{\omega} a}\left[\frac{i}{2}\left(\frac{k_{2}^{\omega}}{k_{1}^{\omega}}-\frac{k_{1}^{\omega}}{k_{2}^{\omega}}\right) \sin k_{2}^{\omega} b\right]
\end{aligned}
$$

and the translation matrix eigenvalue is

$$
\exp \left(i K_{\mathrm{TE}}^{\omega} \Lambda\right)=\exp \left\{i \cos ^{-1}\left[\operatorname{Re}\left(A_{\mathrm{TE}}^{\omega}\right)\right]\right\} \text {. }
$$

Finally, as the waveguide must satisfy the mode dispersion equation at $\omega$

$$
\frac{1}{k_{\mathrm{c}}^{\omega}} \cot \left(\frac{k_{\mathrm{c}}^{\omega} t_{\mathrm{c}}}{2}\right)-\frac{i}{k_{1}^{\omega}} \frac{e^{i K_{\mathrm{TE}}^{\omega} \Lambda}-A_{\mathrm{TE}}^{\omega}+B_{\mathrm{TE}}^{\omega}}{e^{i K_{\mathrm{TE}}^{\omega} \Lambda}-A_{\mathrm{TE}}^{\omega}-B_{\mathrm{TE}}^{\omega}}=F(\omega, \beta)=0 .
$$

Note that (16) depends only on the core thickness, via (10)(15). Since $t_{\mathrm{c}}$ is a real quantity, (16) can easily be solved using numerical root-finding methods.

\section{A. Examples}

To demonstrate the concept of the waveguide discussed here we utilize the $\mathrm{GaAs} / \mathrm{Al}_{x} \mathrm{Ga}_{1-x}$ As material system, which has been studied extensively for frequency conversion due to its mature fabrication technology and large nonlinear coefficient at the half bandgap [25]. To calculate material indices, we use the refractive index dispersion model of Adachi [26]. Fig. 5(a) shows contours of the phase-matching core thickness for a fundamental wavelength of $1550 \mathrm{~nm}$ over a range of core and cladding $\mathrm{Al}$ concentrations. The $\mathrm{Al}$ fraction in the cladding layers is initially chosen to be symmetric about that of the core, i.e., $x_{\text {clad }}=x_{\mathrm{c}} \pm \Delta x_{\text {clad }} / 2$. Al fractions $x_{\mathrm{c}}$ and $\Delta x_{\text {clad }}$ are used instead of material indices $n_{\mathrm{c}}$ and $\Delta n_{\text {clad }}$ as the latter are variable due to material dispersion between the fundamental and $\mathrm{SH}$ wavelengths. Note that the range of $x_{\text {clad }}$ and $\Delta x_{\text {clad }}$ is chosen such that $0.15 \leq x \leq 1$ to ensure that the SH photon energy exceeds the bandgap energy of $\mathrm{Al}_{x} \mathrm{Ga}_{1-x}$ As. Fig. 5(b) shows contours of the phase-matched effective indices. The calculated effective indices were verified by solving the waveguides using the well-known transfer matrix method [27], which makes no a priori assumption on the waveguiding mechanism (BRW or TIR).

Fig. 5(a) and (b) shows that phase-matched solutions exist over a wide range of core and cladding materials, subject to the constraint (9). This allows us to independently optimize other waveguide properties such as SHG conversion efficiency and dispersion, as discussed in Section IV. Of course, $x_{\mathrm{c}}$ need not be at the midpoint of $x_{\text {clad }}$. The relative aluminum fractions among the core and cladding all represent degrees of freedom by which the waveguide properties can be optimized.

\section{WAVEGUide PROPERTIES With Respect TO PHASE MATCHING}

Although qualitatively the simultaneous solution of both modes to achieve phase matching has been proven in Section III it is still not clear quantitatively what would be the figures of merit for such phase-matching techniques. Issues such as dispersion of the fundamental and SH modes, and phase reversal of the BRW mode, etc., would all reduce the overall conversion efficiency. In this section we shall discuss these figures of merit and explore waveguide designs to optimize them. 


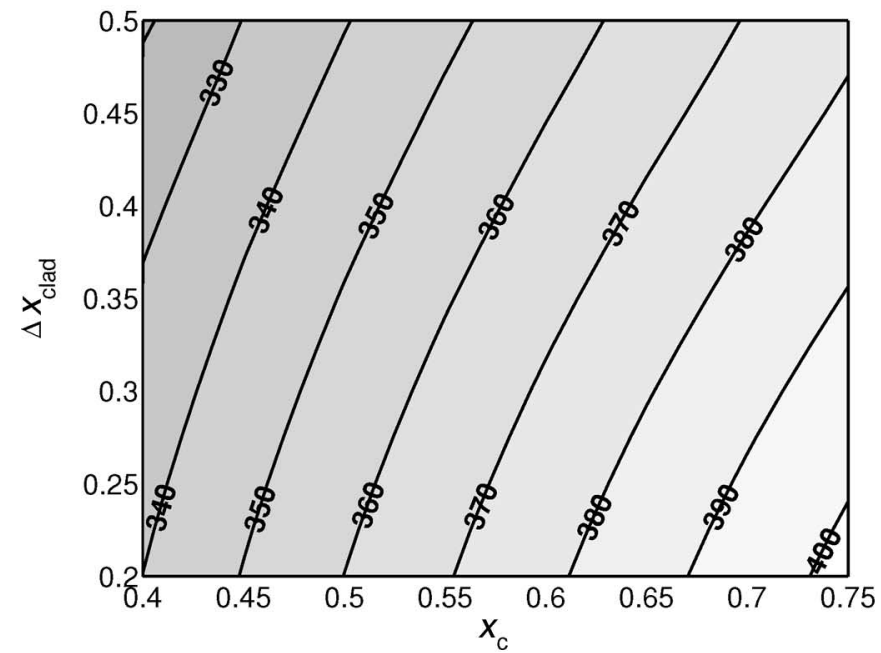

(a)

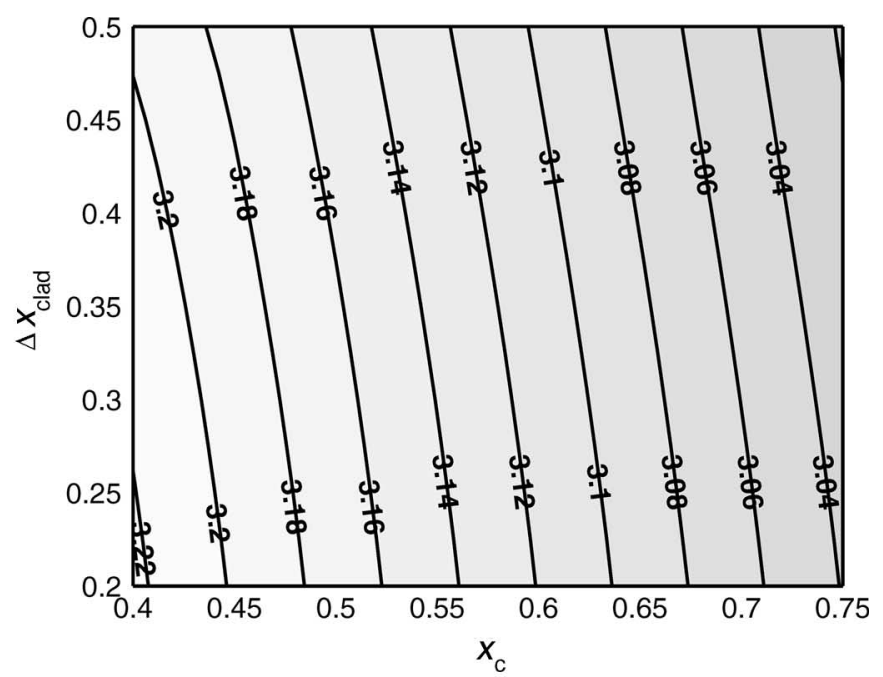

(b)

Fig. 5. (a) Core thickness $[\mathrm{nm}]$ required for phase matching and (b) resulting phase-matched effective index over a range of $x_{\mathrm{C}}$ and $\Delta x_{\mathrm{clad}}\left(x_{\mathrm{clad}}\right.$ symmetric about $x_{\mathrm{C}}$ ).

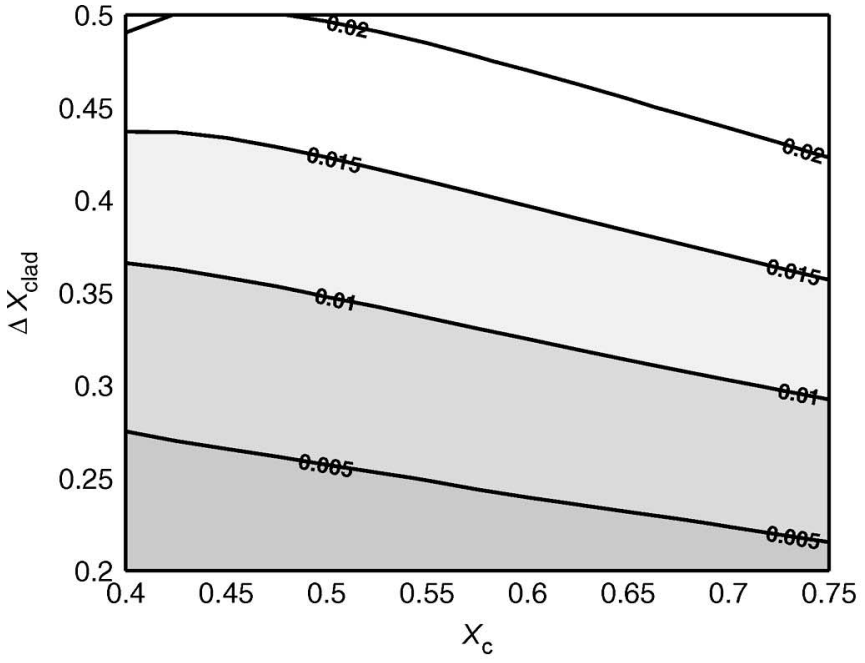

(a)

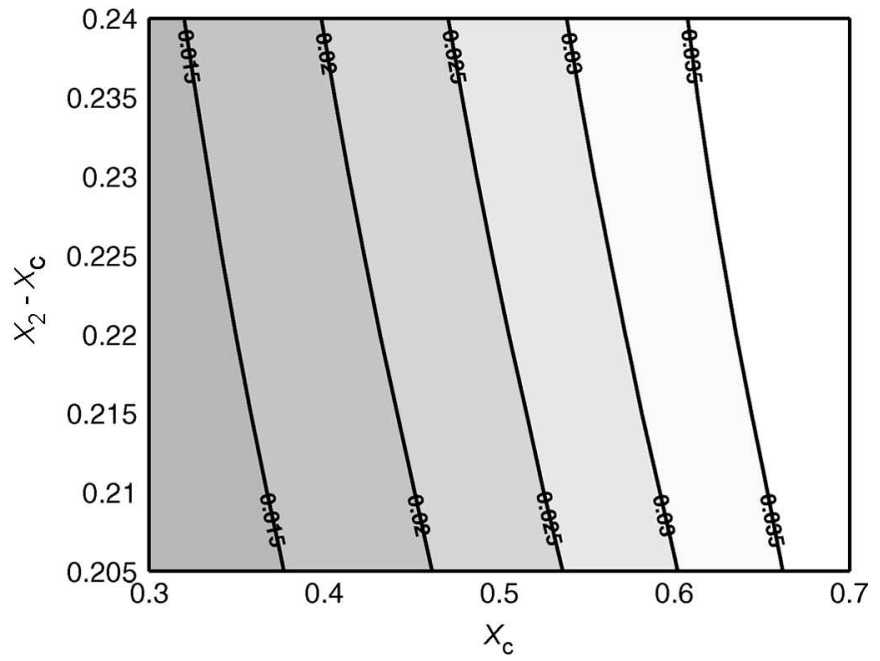

(b)

Fig. 6. (a) $\left(n_{\text {eff }}\right)^{-3}\left(t_{\text {eff }}\right)^{-1}\left[\mu \mathrm{m}^{-1}\right]$ over a range of $x_{\mathrm{c}}$ and $\Delta x_{\text {clad }}\left(x_{\text {clad }}\right.$ symmetric about $\left.x_{\mathrm{c}}\right)$. (b) $\left(n_{\text {eff }}\right)^{-3}\left(t_{\text {eff }}\right)^{-1}\left[\mu \mathrm{m}^{-1}\right]$ over a range of $x_{\mathrm{c}}$ and $x_{2}-x_{\mathrm{c}}$.

\section{A. Nonlinear Coupling Efficiency}

The efficiency with which energy is coupled between the modes depends on the spatial overlap between the induced dielectric polarization at $2 \omega, P^{2 \omega}(x)=\varepsilon_{0} d(x)\left[E^{\omega}(x)\right]^{2}$, and the SH mode, $E^{2 \omega}(x)$, where $\varepsilon_{0}$ is the free-space permittivity. We can thus define a nonlinear coupling efficiency $\eta$ which is inversely proportional to $\left(n_{\text {eff }}\right)^{3}$ and to an effective SHG width [28]

$$
\begin{aligned}
\eta & =n_{\mathrm{eff}}^{-3} \frac{1}{t_{\mathrm{SHG}}^{\mathrm{eff}}} \\
& =n_{\mathrm{eff}}^{-3} \frac{\left[\int_{-\infty}^{\infty}\left[E^{2 \omega}(x)\right]^{*} d^{\prime}(x)\left[E^{\omega}(x)\right]^{2} d x\right]^{2}}{\left[\int_{-\infty}^{\infty}\left[E^{\omega}(x)\right]^{2} d x\right]^{2}\left[\int_{-\infty}^{\infty}\left[E^{2 \omega}(x)\right]^{2} d x\right]}
\end{aligned}
$$

where $d^{\prime}(x)=d(x) / d_{\max }$ is the SHG coefficient, constant across each layer of the waveguide, and $d_{\max }$ is the max- imum SHG coefficient in the structure. The higher effective indices of $\mathrm{GaAs}$ waveguides in comparison to $\mathrm{LiNbO}_{3}$ are more than compensated for by the difference in nonlinear optic coefficient. A geometry-independent figure of merit to compare SHG in two materials is $d^{2} /\left(n_{\mathrm{eff}}\right)^{3}$ [29]. Using $d_{14 \text { (AlGaAs) }}=$ $150 \mathrm{pm} / \mathrm{V}, d_{33 \text { (LiNbO3) }}=32.2 \mathrm{pm} / \mathrm{V}[30], n_{\mathrm{LiNbO} 3}=2.25$, and the effective indices for the GaAs waveguides shown in Fig. 5(b) it is seen that GaAs is roughly 8.25 times more efficient than $\mathrm{LiNbO}_{3}$ for identical effective SHG widths. It should be noted, however, that there is still a great deal of discrepancy in the literature as to the absolute value of the nonlinear coefficient of $\mathrm{AlGaAs}$, particularly as a function of $\mathrm{Al}$ fraction and close to the half bandgap.

Contours of $\eta$, corresponding to the same waveguide structures as in Fig. 5(a), are shown in Fig. 6(a). It is assumed here that $d$ is approximately equal in all regions of the guide, i.e., 


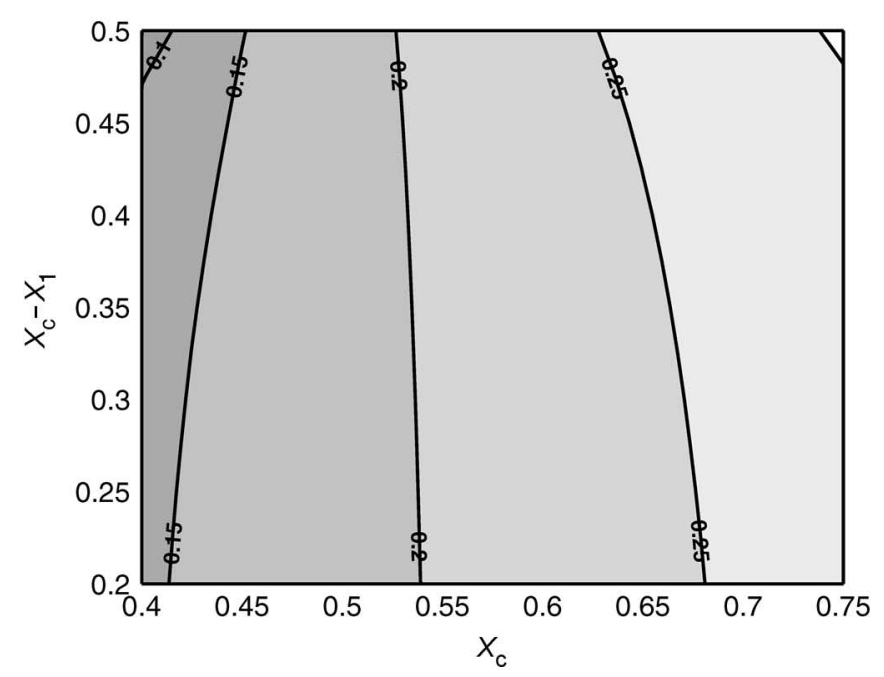

(a)

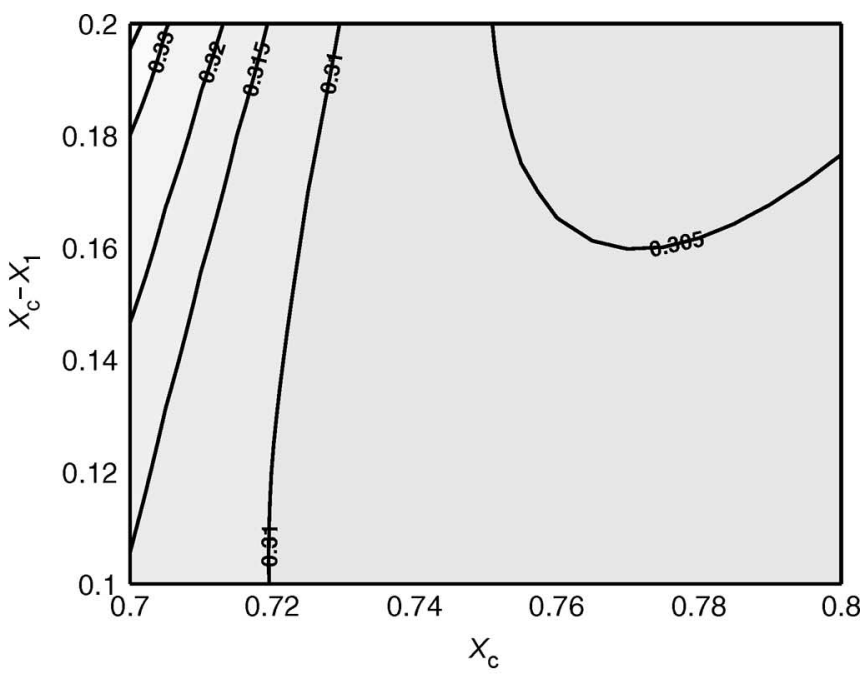

(b)

Fig. 7. (a) Spectral bandwidth (FWHM) for a 1-cm device length over a range of $x_{\mathrm{c}}$ and $\Delta x_{\text {clad }}$ ( $x_{\text {clad }}$ symmetric about $x_{\mathrm{c}}$ ). (b) Spectral bandwidth (FWHM) for a $1-\mathrm{cm}$ device length over a range of $x_{\mathrm{c}}$ and $x_{\mathrm{c}}-x_{1}\left(x_{2}=1\right)$.

$d^{\prime}(x)=1$. The figure indicates that the coupling coefficient can be increased by using large values of $\Delta x_{\text {clad }}$. This can be explained by the oscillation of the $\mathrm{SH}$ mode phase in the cladding as can be seen in Fig. 3. For the efficiency to be maximized, one intuitive means is to maximize the core confinement factor of both modes, which requires a large index difference in the cladding [22]. In Fig. 6(b), $x_{1}$ is set to 0.15 , while $x_{\mathrm{c}}$ and $x_{2}-x_{\mathrm{c}}$ are varied. A significant increase in $\eta$ can be seen for larger values of $x_{\mathrm{c}}$ (lower core index). The optimized value of $\eta \approx 0.04$ is very similar to that obtained in a $\mathrm{TE}_{0}^{\omega}-\mathrm{TE}_{2}^{2 \omega}$ mode dispersion phase matching configuration (normalized by effective index) in [28]. The efficiency can be further maximized by various means, given the versatility afforded by the BRW. These means are discussed in Section VI.

\section{B. Dispersion}

The wavelength dependence of effective index is of great importance in SHG. Waveguides that are phase matched at a nominal wavelength, but for which the dispersion differs between the fundamental and SH wavelengths, will maintain coherence over a finite spectral bandwidth. Furthermore, when ultrashort pulses are used, a mismatch between the group velocities at the two interacting frequencies causes the pulses to separate in time (pulse walkoff), reducing their temporal overlap and hence lowering conversion efficiency [29]. Finally, pulse broadening due to group velocity dispersion (GVD) reduces conversion efficiency by reducing the peak power of the pulses.

To estimate the spectral bandwidth of the structure, we adopt the criterion suggested by Fejer et al. [31]. For imperfect phase matching over a device length $L$, SHG conversion efficiency varies as $[\sin (\Delta L) /(\Delta L)]^{2}$, where $2 \Delta=\beta^{2 \omega}-\beta^{\omega}$. This factor is reduced by $3 \mathrm{~dB}$ when $\Delta L=0.4429 \pi$, allowing us to define a full-width at half-maximum (FWHM) bandwidth around the fundamental wavelength for a nominally phase-matched struc- ture with known length $L_{D}$ :

$$
\Delta \lambda_{\mathrm{FWHM}}=\frac{0.4429 \lambda}{L_{D}}\left(\left|\frac{\partial n_{\mathrm{eff}}^{\omega}}{\partial \lambda}-\frac{1}{2} \frac{\partial n_{\mathrm{eff}}^{2 \omega}}{\partial \lambda}\right|\right)^{-1}
$$

where $\lambda$ is the fundamental wavelength and the derivatives, which include both material and waveguide dispersion, are evaluated at their respective wavelengths. Here, dispersion terms higher than second order have been neglected. Fig. 7(a) shows the contours of spectral bandwidth, for the waveguide structures shown in Fig. 5(a), and a device length of $1 \mathrm{~cm}$. Bandwidth is seen to increase with $x_{\mathrm{c}}$, which can be attributed to the reduction in material dispersion of AlGaAs as the bandgap energy moves away from the photon energy. In Fig. 7(b), bandwidth is shown for $x_{2}=1.0$ over a range of $x_{\mathrm{c}}$ and $\left(x_{c}-x_{1}\right)$. Bandwidth increases to about $0.34 \mathrm{~nm}$ at $\left\{x_{1}, x_{\mathrm{c}}, x_{2}\right\}=\{0.5,0.7,1.0\}$, until the parameter range becomes limited by the existence of phase-matched solutions to the mode dispersion equations, subject to (9). This bandwidth corresponds well with those measured by Rao et al. [7] (0.5-1.0 nm over a $3 \mathrm{~mm}$ length) using modal phase matching in AlGaAs, and with those calculated by Fejer et al. [31] ( $\sim 3 \mathrm{~nm}$ over a $1 \mathrm{~mm}$ length) in PPLN.

Optical pulses travel with a group velocity

$$
v_{g}=\frac{\partial \omega}{\partial \beta}=c\left(n_{\mathrm{eff}}-\lambda \frac{\partial n_{\mathrm{eff}}}{\partial \lambda}\right)^{-1}
$$

which can be evaluated by implicitly differentiating the mode dispersion equation (2)

$$
v_{g}=-\left[\frac{\partial F}{\partial \beta}\right]_{\omega}\left[\frac{\partial F}{\partial \omega}\right]_{\beta}^{-1} .
$$

The group velocity mismatch (GVM) is equal to the difference between inverse group velocities, which in the case of nominal phase matching becomes

$$
\operatorname{GVM}=\left|\frac{1}{v_{g}^{\omega}}-\frac{1}{v_{g}^{2 \omega}}\right|=\frac{\lambda}{c}\left|\left(\frac{\partial n_{\mathrm{eff}}^{\omega}}{\partial \lambda}-\frac{1}{2} \frac{\partial n_{\mathrm{eff}}^{2 \omega}}{\partial \lambda}\right)\right| .
$$




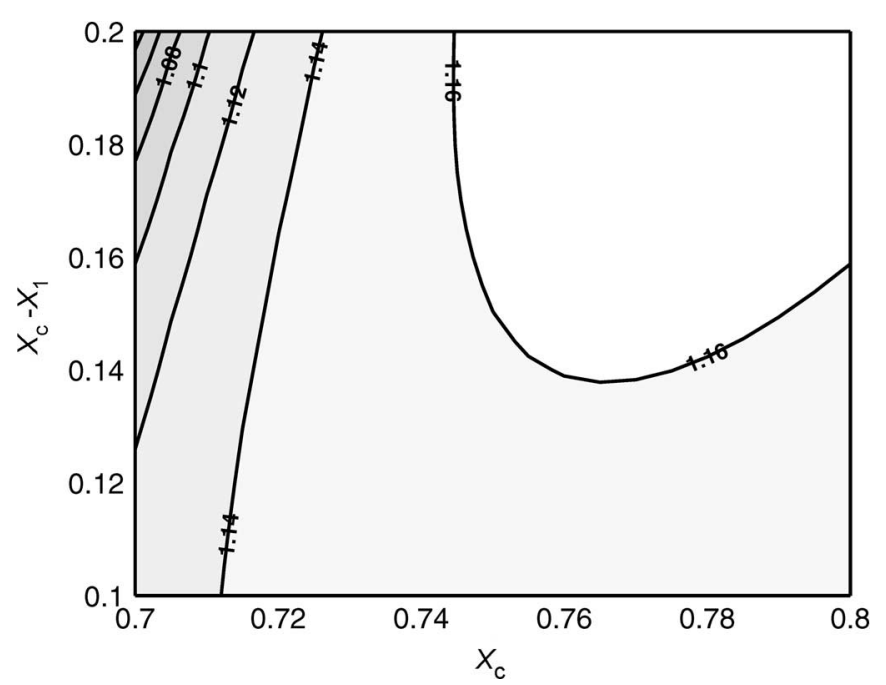

Fig. 8. Group velocity mismatch $(\mathrm{ps} / \mathrm{mm})$ over a range of $x_{\mathrm{c}}$ and $x_{c}-$ $x_{1}\left(x_{2}=1\right)$.

Comparing (21) with (18), it is clear that the spectral bandwidth and GVM are optimized simultaneously, as both require minimization of the difference in dispersion between the two modes. Fig. 8 shows GVM over the optimized parameter range used in Fig. 7(b). GVM decreases to a value of $\approx 1.03 \mathrm{ps} / \mathrm{mm}$ at $\left\{x_{1}, x_{\mathrm{c}}, x_{2}\right\}=\{0.5,0.7,1.0\}$. This GVM allows pulses at $\omega$ of width $\tau_{0}^{\mathrm{GVM}}>10$ ps to propagate through a waveguide of $1 \mathrm{~cm}$ length without appreciable reduction in SHG efficiency due to pulse walkoff. To enable phase matching over larger bandwidths and for shorter pulses, hence higher peak intensities, optimization can be carried out; in Section VI, methods of further reducing GVM are discussed.

GVD for a device length $L_{D}$ is quantified by a characteristic pulsewidth [29]

$$
\tau_{0}^{\mathrm{GVD}}=\sqrt{8 L_{D} \frac{\partial^{2} \beta}{\partial \omega^{2}}}
$$

below which the pulse will experience appreciable broadening. The term $\partial^{2} \beta / \partial \omega^{2}$ can be evaluated by further differentiation of (20). Fig. 9(a) shows $\partial^{2} \beta / \partial \omega^{2}$ (in units of $\mathrm{fs}^{2} / \mu \mathrm{m}$ ) for the fundamental mode, for the range of parameters used in Fig. 5(a), and $L_{D}=1 \mathrm{~cm}$. The average value of $0.75 \mathrm{fs}^{2} / \mu \mathrm{m}$ corresponds to $\tau_{0}^{\mathrm{GVD}} \sim 250 \mathrm{fs}$, which is negligible in comparison to $\tau_{0}^{\mathrm{GVM}}$.

GVD at the SH wavelength is shown in Fig. 9(b). Values of several hundred $\mathrm{fs}^{2} / \mu \mathrm{m}$ are found, as can be expected for a resonant device operating in the middle of the stopband. Fig. 9(c) shows GVD for $x_{2}=1.0$, over a range of $x_{\mathrm{c}}$ and $\left(x_{c}-x_{1}\right)$, which decreases with the index difference in the cladding (weak resonance). A GVD of $\sim 40 \mathrm{fs}^{2} / \mu \mathrm{m}$ in this region corresponds to $\tau_{0}^{\mathrm{GVD}}<2$ ps over $1 \mathrm{~cm}$, again small in comparison to $\tau_{0}^{\mathrm{GVM}}$. Thus, a tradeoff exists between GVD and SHG conversion efficiency, which requires large index difference in the cladding (minimization of propagation loss also requires a large index difference, as is demonstrated in Section IV-C). From these calculations, it can be seen that the pulses of duration greater than $10 \mathrm{ps}$ can be phase matched over a distance of $1 \mathrm{~cm}$ without appreciable reduction in SHG efficiency.

\section{Propagation Loss}

The preceding derivations assumed that the cladding consists of an infinite number of periods, such that the propagation loss due to leakage of the waveguide is zero. For practical devices, there will be a finite number of cladding periods. The leakage in such devices can be estimated using a ray-optics approach, as in [32]. We assume that the loss is low enough, that the propagation constant $\beta$ is essentially real, and that there is negligible perturbation to the unit cell translation matrix formulation described in Section II.

In the ray-optics picture, the angle of incidence between core and cladding is given by

$$
\theta_{i}=\sin ^{-1}\left(\frac{n_{\text {eff }}}{n_{\mathrm{c}}}\right)
$$

and the number of reflections per unit length is $1 /\left(t_{\mathrm{c}} \tan \theta_{i}\right)$. The reflection coefficient at the interface between the core and finite cladding can be calculated using the transfer matrix formalism of Chilwell and Hodgkinson [27]. Here, the field amplitudes in the $j$ th layer are represented by a matrix

$$
\mathbf{M}_{j}=\left(\begin{array}{cc}
\cos \Phi_{j} & \frac{-i}{\gamma_{j}} \sin \Phi_{j} \\
-i \gamma_{j} \sin \Phi_{j} & \cos \Phi_{j}
\end{array}\right)
$$

where

$$
\gamma_{j}=\frac{1}{\left(n_{j}\right)^{2 \rho}} \sqrt{n_{j}^{2}-n_{\mathrm{eff}}^{2}}, \quad \rho= \begin{cases}0 & (\mathrm{TE}) \\ 1 & (\mathrm{TM})\end{cases}
$$

and

$$
\Phi_{j}=k_{j} t_{j}
$$

$\left(t_{j}=a, b\right)$ is the phase thickness of the layer. The transfer matrix for a stack of films is given by the product of these matrices

$$
\mathbf{M}=\prod_{j=1}^{J} \mathbf{M}_{j} .
$$

$$
\begin{aligned}
\mathbf{M}_{\text {clad }} & =\left(\mathbf{M}_{1} \mathbf{M}_{2}\right)^{\mathrm{N}} \\
& =\left(\begin{array}{cc}
\cos \Phi_{1} \cos \Phi_{2}-\left(\frac{\gamma_{2}}{\gamma_{1}}\right) \sin \Phi_{1} \sin \Phi_{2} & -i\left(\frac{1}{\gamma_{1}} \sin \Phi_{1} \cos \Phi_{2}+\frac{1}{\gamma_{2}} \cos \Phi_{1} \sin \Phi_{2}\right) \\
-i\left(\gamma_{1} \sin \Phi_{1} \cos \Phi_{2}+\gamma_{2} \cos \Phi_{1} \sin \Phi_{2}\right) & \cos \Phi_{1} \cos \Phi_{2}-\left(\frac{\gamma_{1}}{\gamma_{2}}\right) \sin \Phi_{1} \sin \Phi_{2}
\end{array}\right)^{\mathrm{N}} \\
& \equiv\left(\begin{array}{ll}
m_{11}^{\mathrm{N}} & m_{12}^{\mathrm{N}} \\
m_{21}^{\mathrm{N}} & m_{22}^{\mathrm{N}}
\end{array}\right) .
\end{aligned}
$$




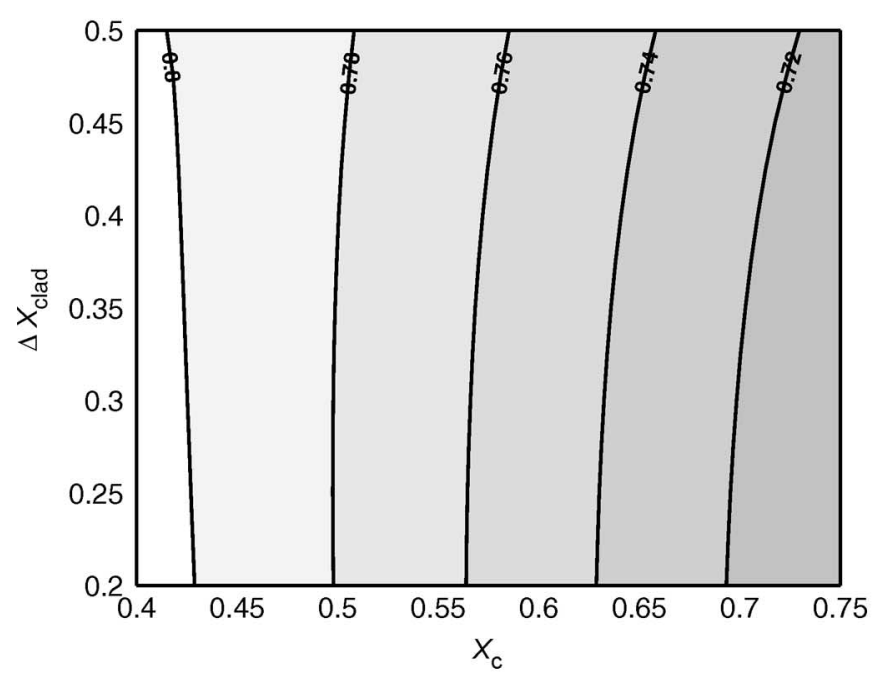

(a)

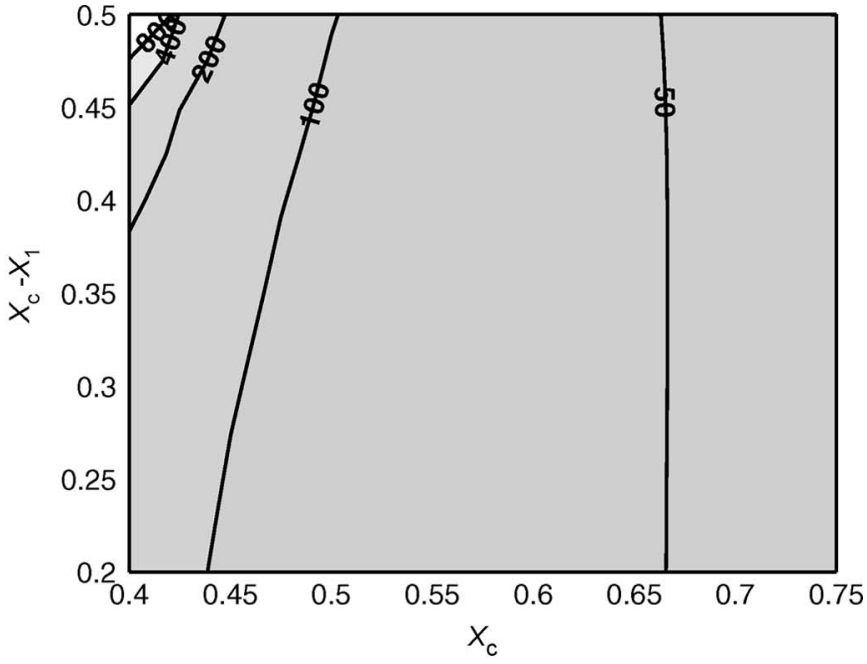

(b)

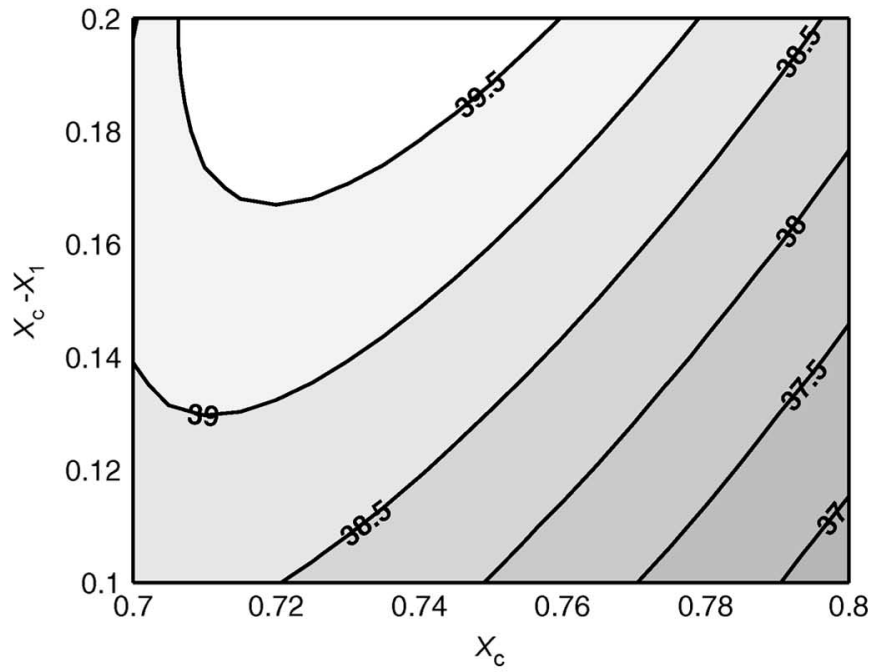

(c)

Fig. 9. Group velocity dispersion $\partial^{2} \beta / \partial \omega^{2}$ (fs ${ }^{2} / \mu \mathrm{m}$ ) of (a) fundamental mode over a range of $x_{\mathrm{c}}$ and $\Delta x_{\text {clad }}\left(x_{\text {clad }}\right.$ symmetric about $x_{\mathrm{c}}$ ), (b) SH mode over a range of $x_{\mathrm{c}}$ and $\Delta x_{\mathrm{clad}}$, and (c) SH mode over a range of $x_{\mathrm{c}}$ and $x_{\mathrm{c}}-x_{1}\left(x_{2}=1\right)$.

Thus, the cladding of an N-period BRW has transfer matrix shown by (28), at the bottom of the previous page. For the QtW-BRW, $\Phi_{j}=\pi / 2$, and (28), reduces to

$$
\mathbf{M}^{\lambda / 4}=(-1)^{\mathrm{N}}\left(\begin{array}{cc}
\left(\frac{\gamma_{2}}{\gamma_{1}}\right)^{\mathrm{N}} & 0 \\
0 & \left(\frac{\gamma_{1}}{\gamma_{2}}\right)^{\mathrm{N}}
\end{array}\right)
$$

In the general case, the unimodularity of $\mathbf{M}_{1} \mathbf{M}_{2}$ can be exploited to calculate (28), using the Chebyshev identity [33]

$$
\begin{aligned}
& \left(\begin{array}{ll}
m_{11} & m_{12} \\
m_{21} & m_{22}
\end{array}\right)^{\mathrm{N}} \\
& =\left(\begin{array}{cc}
m_{11} U_{\mathrm{N}-1}-U_{\mathrm{N}-2} & m_{12} U_{\mathrm{N}-1} \\
m_{21} U_{\mathrm{N}-1} & m_{22} U_{\mathrm{N}-1}-U_{\mathrm{N}-2}
\end{array}\right)
\end{aligned}
$$

where

$$
\begin{aligned}
U_{\mathrm{N}} & =\frac{\sin (\mathrm{N}+1) \zeta}{\sin \zeta} \\
\zeta & =\cos ^{-1}\left[\frac{m_{11}+m_{22}}{2}\right] .
\end{aligned}
$$

The reflection coefficient can then be calculated from the transfer matrix [27] as

$$
r=\frac{\gamma_{\mathrm{c}} m_{11}^{\mathrm{N}}+\gamma_{\mathrm{c}} \gamma_{\mathrm{sub}} m_{12}^{\mathrm{N}}-m_{21}^{\mathrm{N}}-\gamma_{\mathrm{sub}} m_{22}^{\mathrm{N}}}{\gamma_{\mathrm{c}} m_{11}^{\mathrm{N}}+\gamma_{\mathrm{c}} \gamma_{\mathrm{sub}} m_{12}^{\mathrm{N}}+m_{21}^{\mathrm{N}}+\gamma_{\mathrm{sub}} m_{22}^{\mathrm{N}}}
$$

where $\gamma_{\mathrm{c}}$ and $\gamma_{\mathrm{sub}}$ are defined analogously to (25) for the core and substrate. For the QtW-BRW, from (29),

$$
r=\frac{\gamma_{\mathrm{c}}\left(\frac{\gamma_{2}}{\gamma_{1}}\right)^{\mathrm{N}}-\gamma_{\mathrm{sub}}\left(\frac{\gamma_{1}}{\gamma_{2}}\right)^{\mathrm{N}}}{\gamma_{\mathrm{c}}\left(\frac{\gamma_{2}}{\gamma_{1}}\right)^{\mathrm{N}}+\gamma_{\mathrm{sub}}\left(\frac{\gamma_{1}}{\gamma_{2}}\right)^{\mathrm{N}}} .
$$

As $\gamma_{1}>\gamma_{2}, \lim _{N \rightarrow \infty} r=-1$, as noted in Section II. 


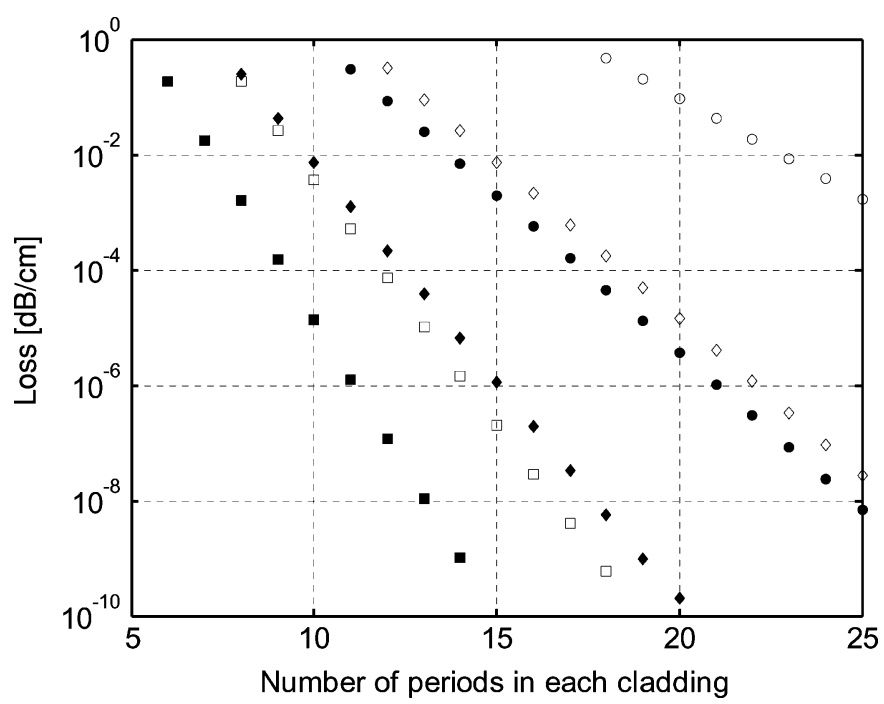

Fig. 10. Propagation loss as a function of the number of cladding periods. Filled markers: BRW mode. Hollow markers: TIR mode. $x_{\mathrm{c}}=0.5, \Delta x_{\mathrm{clad}}=$ $0.3(\bigcirc), 0.4(\diamond), 0.5(\square)$.

The power remaining after each reflection is $|r|^{2}$. We can now calculate the propagation loss using (23)-(34)

$$
\text { Loss }[\mathrm{dB} / \mathrm{cm}]=\frac{-\ln (|r|)}{5 t_{\mathrm{c}} \tan \theta_{i}} .
$$

Plots of propagation loss versus $\mathrm{N}$ for various values of $\Delta x_{\text {clad }}$ are shown in Fig. 10. As expected, loss decreases exponentially with $\mathrm{N}$ and is lower for larger $\Delta x_{\text {clad. }}$. It can be seen that for a few periods, propagation loss due to leakage is negligible in comparison to the expected scattering losses. Therefore, it can be seen that for the quarter-wave case whilst working in the middle of the Bragg bandgap, it is acceptable to use the theory of infinite cladding layers to approximate a finite cladding.

\section{Phase-Matching Tunability}

Many of the applications that rely on second order nonlinearities also depend on the capability of tuning the nonlinearity, and hence the phase matching, for them to be useful. Examples include tunable difference frequency generation for optical communications, tunable OPOs, and tunable intensitydependent switches that rely on cascaded second order nonlinearities. Temperature and angle tuning have been the two most popular tuning methods for the PPLN technology. Angle tuning is not applicable to guided wave devices and was only useful for collimated beam configurations such as with PPLN. While temperature tuning is applicable for devices such as those studied here, the technique suffers from numerous unattractive features that make it undesirable. Performance stability, repeatability, thermal time constants, and setup complexity are some of these drawbacks. In addition, as the devices studied here are implemented in semiconductors, it is advantageous to make use of the various tuning methods offered by these materials. Techniques such as electro-optic and carrier induced tuning lend themselves to robust, low-power, and high-precision tuning in integrated photonic device environments.
Tuning the modes of BRWs to remain phase matched with TIR modes is not straightforward, as a significant perturbation of the cladding will cause a deviation from the quarter-wave condition, and may in fact shift the BRW mode entirely out of the stopband, increasing the mode loss. One of the most promising means of tuning such structures is to restrict the refractive index change to the core layer. This is because such effects will be similar to tuning the material thickness and hence will have minimal effect on the other waveguiding properties as well as provide continuous tuning. The examples mentioned in this section intend to provide an order-of-magnitude estimate of the tuning range; detailed modeling of the tuning mechanisms will appear in a future work.

\section{A. Carrier Tuning}

Carrier tuning relies on the dependence of the semiconductor refractive index on the concentration of free carriers. The most common route to controlling the carrier concentration is through current injection. A doped structure with a p-i-n doping profile, where the intrinsic region is the volume that needs to be tuned, is usually used for this purpose. As the structure is put in forward bias, current flows across the depletion region which coincides primarily with the intrinsic region. The technique has been used to tune filters [34] and tunable lasers [35]. In the waveguide structure investigated here, if we $\mathrm{p}$-and $\mathrm{n}$-dope the upper and lower cladding regions, respectively, an appreciable carrier density in the core will change the core index, thus shifting the effective index of both the BRW and TIR modes and altering the phase-matching wavelength.

The effect of a given carrier concentration on the refractive index of GaAs has been studied by numerous authors [36], [37]. A full model calculates the spectral shift of absorption due to bandfilling (Burstein-Moss effect), bandgap shrinkage, and free-carrier absorption (plasma effect), with subsequent evaluation of the index shift by Kramers-Kronig analysis [38], and shows a roughly linear decrease of $n$ with carrier density $N$ for $N>10^{18} \mathrm{~cm}^{-3}$, with $d n / d N \approx-10^{-20} \mathrm{~cm}^{3}$ [36]. In this way, index changes up to -0.1 can be readily achieved. In Fig. 11, the phase-matching fundamental wavelength is shown over a range of carrier concentrations for a waveguide with $\left\{x_{1}, x_{\mathrm{c}}, x_{2}\right\}=\{0.3,0.5,0.7\}$. Wavelength tunability over several tens of nanometers can be accomplished using carrier densities of $10^{19} \mathrm{~cm}^{-3}$, which is not uncommon to achieve in active photonic devices. This tuning range will ultimately be limited by the increased absorption of the tuned SH mode as it approaches the core bandgap. However, the effects of the excess free-carrier absorption are not included here.

\section{B. Electro-Optic Tuning}

Unlike carrier tuning, which takes place in a forward biased p-n junction, electro-optic (EO) tuning takes place in a reverse biased p-n junction. In III-V semiconductors, the EO effects available include linear EO, quadratic EO, Franz-Keldysh, or, if quantum wells are present, quantum confined Stark effects [39], [40]. For simplicity and in order to demonstrate the principle, we shall restrict the demonstration here to the linear 


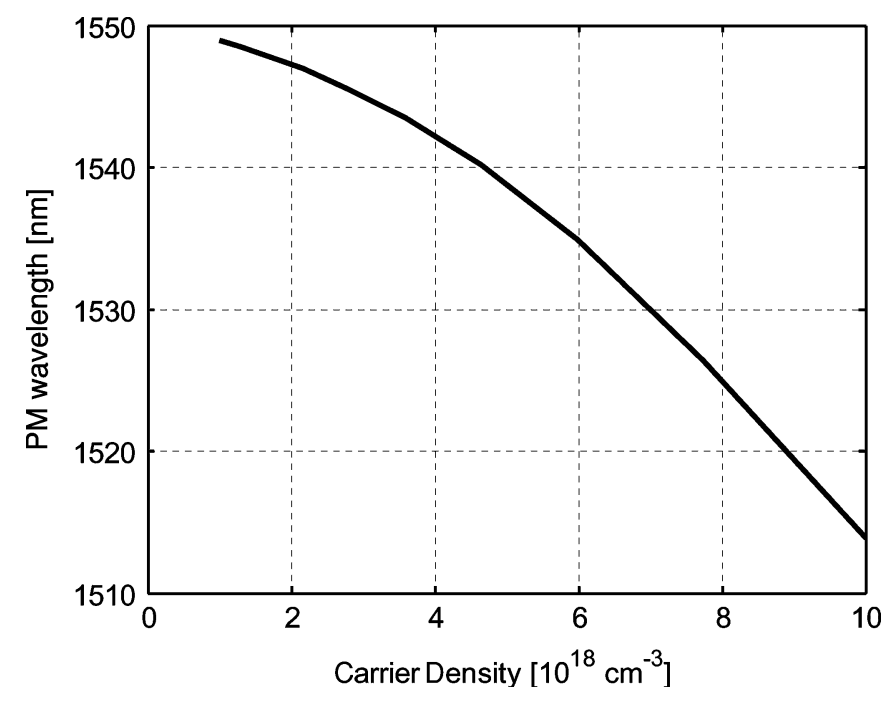

Fig. 11. Example of carrier tuning: phase-matching wavelength versus carrier density.

EO effect; investigation of the other EO tuning methods will occur in a future work. As with carrier tuning, we can design a $\mathrm{p}-\mathrm{i}-\mathrm{n}$ structure, where the intrinsic region is the waveguide core. Thus, in reverse bias, the electric field will fall chiefly across the core (we assume here that the widening of the depletion layer with reverse bias is negligible).

The change in refractive index with applied field $E$ is given by

$$
\Delta n_{\mathrm{c}}=\frac{1}{2} r n_{\mathrm{c}}^{3} E
$$

where $r$ is the linear EO coefficient appropriate to the crystal orientation and field polarization. Extrapolating data from [41], we use $r=-1.5 \mathrm{pm} / \mathrm{V}$ at $1550 \mathrm{~nm}$ and $-1.9 \mathrm{pm} / \mathrm{V}$ at $775 \mathrm{~nm}$. The applied field must be less than the dielectric breakdown field, which is around $300 \mathrm{kV} / \mathrm{cm}$. From (36), the largest index difference achievable using the linear EO effect is $\Delta n_{\mathrm{c}} \approx-0.001$. Fig. 12 shows a tuning curve for a waveguide with $\left\{x_{1}, x_{\mathrm{c}}, x_{2}\right\}=\{0.3,0.5,0.7\}$, with the shift in fundamental phase-matching wavelength with applied voltage shown, due to the small tuning range achieved. The tuning slope is approximately $-29 \mathrm{pm} / \mathrm{V}$, with a maximum wavelength tuning of $-0.29 \mathrm{~nm}$ at $10 \mathrm{~V}$ (which is close to the breakdown voltage for this structure). This fine tuning of the phase-matching wavelength can be used to compensate for drift in the pump wavelength.

\section{ADVANCED DESIGNS}

Further improvements to the design of the BRW/TIR phasematched waveguide can be achieved using advanced optimization techniques. In Section IV, the properties of the waveguide were examined by exploring various contours of parameter space that were expected to provide optimal performance. A more thorough optimization technique might involve the application of evolutionary algorithms to seek out globally optimal waveguide designs depending on the particular device application.

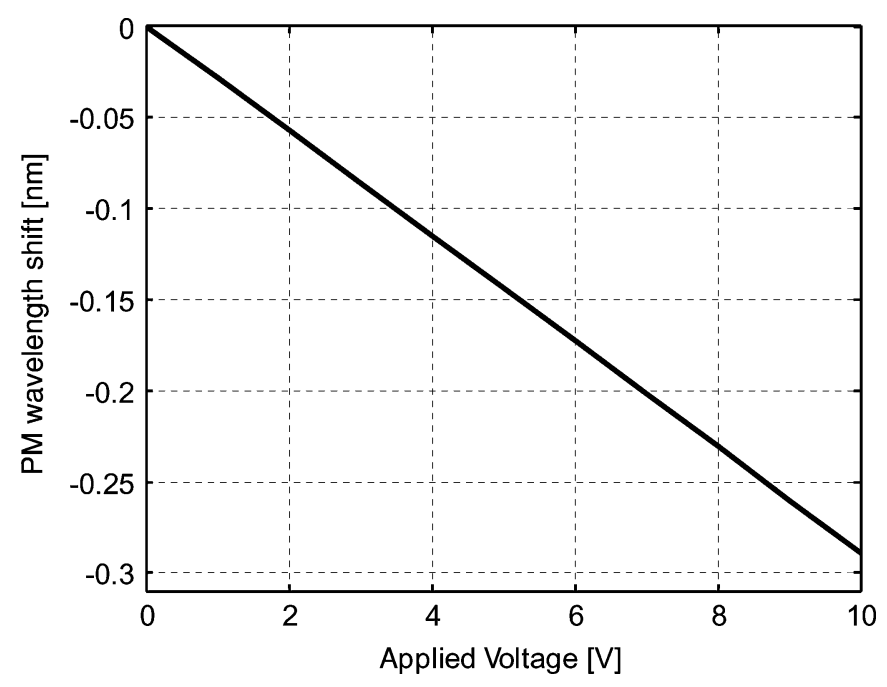

Fig. 12. Example of linear electro-optic tuning: phase-matching wavelength shift versus applied voltage.

Many of the waveguide properties can be improved upon by deviating from the structure shown in Fig. 2. The SHG conversion efficiency of this device is limited by the oscillatory behavior of the BRW mode, as seen in Fig. 3. Examining the contribution to effective thickness from cladding period n, it can be shown that

$$
\begin{aligned}
& \left|\int_{n=2 \mathrm{q}}\left[E^{2 \omega}(x)\right]^{*} d^{\prime}(x)\left[E^{\omega}(x)\right]^{2} d x\right| \\
& >\left|\int_{n=2 \mathrm{q}+1}\left[E^{2 \omega}(x)\right]^{*} d^{\prime}(x)\left[E^{\omega}(x)\right]^{2} d x\right|, \quad q=0,1, \ldots
\end{aligned}
$$

and, therefore, each pair of cladding periods contributes

$$
\sum_{n=2 q}^{2 q+1} \int_{n}\left[E^{2 \omega}(x)\right]^{*} d^{\prime}(x)\left[E^{\omega}(x)\right]^{2} d x<0
$$

with respect to the contribution of the core. This problem has been overcome by various authors in $\mathrm{TE}_{0}^{\omega}-\mathrm{TE}_{2}^{2 \omega}$ modal dispersion phase matching by using rotationally twinned domains to reverse the sign of $d^{\prime}$ at the points where the $\mathrm{TE}_{2}^{2 \omega}$ mode changes the sign [28]. While domain reversal in GaAs-AlGaAs has been achieved [11], [12], it has been applied only as a single reversal, patterned in the direction of propagation, to achieve QPM. Reversing the domain between every cladding period would be technologically unfeasible and would create problems for current injection into the core. However, a single domain reversal at one core/cladding interface is possible and would allow the contributions of each cladding to cancel out. The integrand of the numerator of (17) is plotted in Fig. 13 to illustrate this point. In this case, the problem is reduced to maximizing the overlap of both modes with the core. Fig. 14 shows $\eta$ for this case, using the same waveguide indices as in Fig. 6(b). The efficiency is seen to improve by a factor of 1.5 to 2 over this range. It should be noted that inverting one cladding destroys the symmetry of the induced polarization at $2 \omega$, allowing for 


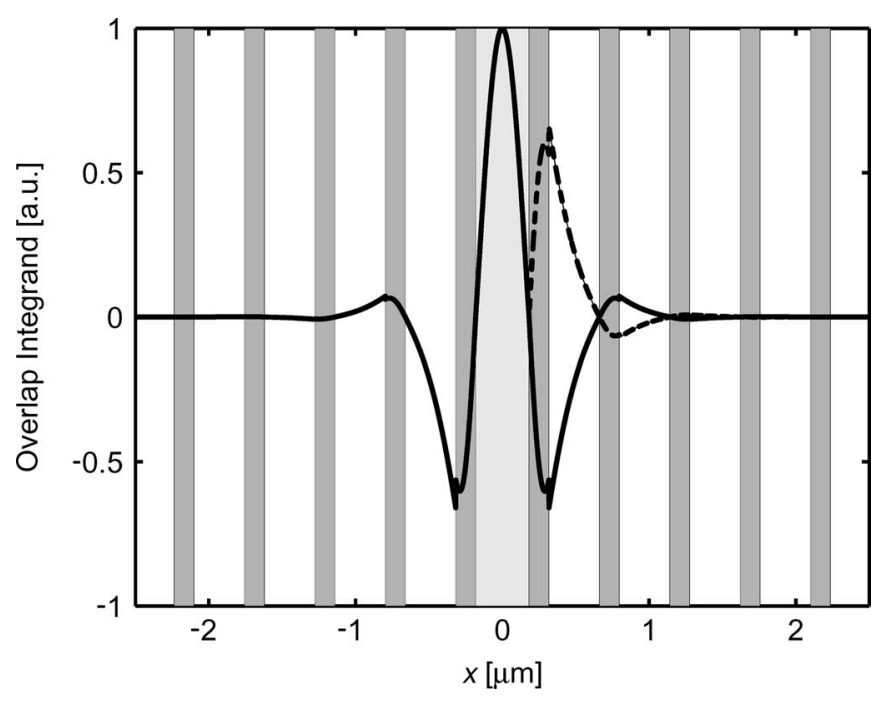

Fig. 13. Integrand of the numerator of (17), showing the effect of domain reversal in the cladding. (Solid line) No domain reversal. (Dashed line) domain reversed in top cladding $\left(x>t_{\mathrm{c}} / 2\right)$.

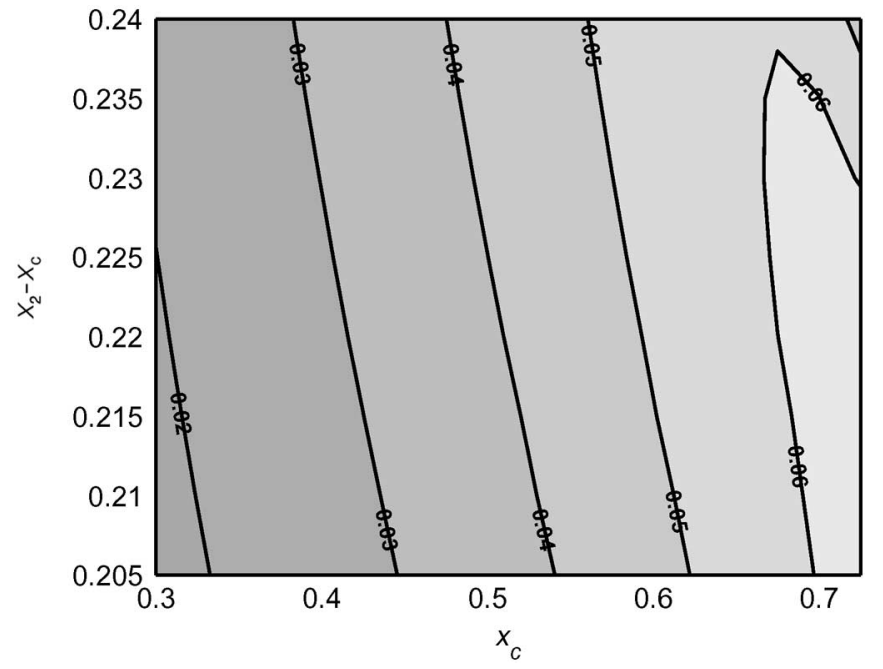

Fig. 14. $\left(n_{\text {eff }}\right)^{-3}\left(t_{\text {eff }}\right)^{-1}\left[\mu \mathrm{m}^{-1}\right]$ over the range of waveguide designs used in Fig. 6(b), with one cladding domain inverted.

coupling to odd BRW modes, which may be useful for type-I phase matching when narrow waveguide cores are used [22].

To avoid the necessity of domain inversion, the index profile of the cladding may be modified in a number of ways. Specifically, the use of a multilayer cladding period is expected to improve spatial overlap of the modes while tailoring the dispersion to improve the spectral properties of the waveguide. Clearly, though, this will require a substantial alteration to the unit cell translation matrix formalism described in Section II.

Finally, it is possible to allow the thicknesses and compositions of each layer to become degrees of design freedom, subject to the constraint that the fundamental and $\mathrm{SH}$ modes experience TIR and Bragg waveguiding (although not necessarily at the quarter-wave condition), respectively. In this case, any number of waveguide properties can be simultaneously optimized. To this end, optimization methods such as the genetic algorithm have been successfully applied to design problems involving stacks of thin films [42], [43].

\section{CONCLUSION}

An innovative method has been introduced to provide phase matching for SHG within a waveguide. The waveguide operates via TIR at the fundamental wavelength and by transverse Bragg reflection at the $\mathrm{SH}$ wavelength. The Bragg waveguide is capable of guiding modes with effective indices lower than the material indices, making it an excellent choice to compensate for the large dispersion of semiconductors near their electronic resonances, where nonlinear effects are enhanced. By forcing the BRW to operate in the center of the stopband, we derived an analytical solution to the phase-matching core thickness for arbitrary core and cladding materials.

Using the AlGaAs material system as an example, we investigated the dispersion, phase-matching bandwidth, and nonlinear coupling efficiency. The applicability of free carrier and EO tuning of the phase-matching wavelength was discussed, with order-of-magnitude estimates given for the tuning range. The waveguide properties compare favorably with many other methods that have been developed for phase matching, and as such, the concept holds great promise to provide efficient monolithically integrated nonlinear elements in semiconductors.

\section{REFERENCES}

[1] W. Ishak, "The future of light: Pervasiveness of photonics in communications, sensors, and life sciences," presented at the Plenary Presentation, SPIE Photonics North, Ottawa, ON, Canada, Sep. 2004.

[2] T. Morioka, "Ultrafast and wideband all-optical processing technologies towards flexible photonic networks," Opt. Rev., vol. 11, no. 3, pp. 153-161, May/Jun. 2004.

[3] A. Bogoni, L. Poti, C. Porzi, M. Scaffardi, P. Ghelfi, and F. Ponzini, "Modeling and measurement of noisy SOA dynamics for ultrafast applications," IEEE J. Sel. Topics Quantum Electron., vol. 10, no. 1, pp. 197-205, Jan./Feb. 2004.

[4] S. Tanzilli, H. De Riedmatten, W. Tittel, H. Zbinden, P. Baldi, M. De Micheli, D. B. Ostrowsky, and N. Gisin, "Highly efficient photon-pair source using periodically poled lithium niobate waveguide," Electron. Lett., vol. 37, no. 1, pp. 26-28, Jan. 2001.

[5] R. V. Roussev, C. Langrock, J. R. Kurz, and M. M. Fejer, "Periodically poled lithium niobate waveguide sum-frequency generator for efficient single-photon detection at communication wavelengths," Opt. Lett., vol. 29, no. 13, (Art. no. 257901), pp. 1518-1520, Jul. 2004.

[6] K. Banaszek, A. Dragan, W. Wasilewski, and C. Radzewicz, "Experimental demonstration of entanglement-enhanced classical communication over a quantum channel with correlated noise," Phys. Rev. Lett., vol. 92, no. 257901, Jun. 2004.

[7] S. V. Rao, K. Moutzouris, and M. Ebrahimzadeh, "Nonlinear frequency conversion in semiconductor optical waveguides using birefringent, moda and quasi-phase-matching techniques," J. Opt. A, vol. 6, no. 6, pp. 569584, Jun. 2004.

[8] M. C. Cardakli, A. B. Sahin, O. H. Adamczyk, A. E. Willner, K R. Parameswaran, and M. M. Fejer, "Wavelength conversion of subcarrier channels using difference frequency generation in a PPLN waveguide," IEEE Photon. Technol. Lett., vol. 14, no. 9, pp. 1327-1329, Sep. 2002.

[9] A. Fiore, S. Janz, L. Delobel, P. van der Meer, P. Bravetti, V. Berger, E. Rosencher, and J. Nagle, "Second-harmonic generation at $\lambda=1.6 \mu \mathrm{m}$ in $\mathrm{AlGaAs} / \mathrm{Al}_{2} \mathrm{O}_{3}$ waveguides using birefringence phase matching," Appl. Phys. Lett., vol. 72, no. 23, pp. 2942-2944, Jun. 1998.

[10] A. S. Helmy, D. C. Hutchings, T. C. Kleckner, J. H. Marsh, A. C. Bryce, J. M. Arnold, C. R. Stanley, J. S. Aitchison, C. T. A. Brown, K. Moutzouris, and M. Ebrahimzadeh, "Quasi phase matching in GaAs-AlAs superlattice waveguides via bandgap tuning using quantum well intermixing," Opt Lett., vol. 25, no. 18, pp. 1370-1373, Sep. 2000. 
[11] S. Koh, T. Kondo, Y. Shiraki, and R. Ito, "GaAs/Ge/GaAs sublattice reversal epitaxy and its applications to nonlinear optical devices," J. Cryst. Growth, vol. 227, pp. 183-192, Jul. 2001.

[12] C. B. Ebert, L. A. Eyres, M. M. Fejer, and J. S. Harris Jr., "MBE growth of antiphase GaAs films using GaAs/Ge/GaAs heteroepitaxy," J. Cryst. Growth, vol. 201/202, pp. 187-193, May 1999.

[13] R. Haidar, N. Forget, and E. Rosencher, "Optical parametric oscillation in micro-cavities based on isotropic semiconductors: A theoretical study," IEEE J. Quantum Electron., vol. 39, no. 4, pp. 569-576, Apr. 2003.

[14] A. S. Helmy, "Phase matching using Bragg reflection waveguides for monolithic nonlinear optics applications," Opt. Express, vol. 14, no. 3, pp. 1243-1252, Feb. 2006.

[15] P. Yeh and A. Yariv, "Bragg reflection waveguides," Opt. Commun., vol. 19, no. 3, pp. 427-430, Dec. 1976

[16] P. Yeh, A. Yariv, and C.-S. Hong, "Electromagnetic propagation in periodic stratified media: I. General Theory," J. Opt. Soc. Amer., vol. 67, no. 4, pp. 428-438, Apr. 1977, and references within.

[17] Y. Sakurai and F. Koyama, "Proposal of tunable hollow waveguide distributed Bragg reflectors," Jpn. J. Appl. Phys., vol. 43, no. 5A, pp. L631L633, 2004.

[18] A. Mizrahi and L. Schachter, "Optical Bragg accelerators," Phys. Rev. E, vol. 70, no. 016505, Jul. 2004.

[19] E. Simova and I. Golub, "Polarization splitter/combiner in high index contrast Bragg reflector waveguides," Opt. Express, vol. 11, no. 25, pp. 3425 3430, Dec. 2003.

[20] C. Wächter, F. Lederer, L. Leine, U. Trutschel, and M. Mann, "Nonlinear Bragg reflection waveguide," J. Appl. Phys., vol. 71, no. 8, pp. 3688-3692, Apr. 1992.

[21] P. M. Lambkin and K. A. Shore, "Nonlinear semiconductor Bragg reflection waveguide structures," IEEE J. Quantum Electron., vol. 27, no. 3 pp. 824-829, Mar. 1991.

[22] B. R. West and A. S. Helmy, "Properties of the quarter-wave Bragg reflection waveguide: Theory,"J. Opt. Soc. Amer. B, Opt. Phys., to be published.

[23] S. G. Johnson, M. Ibanescu, M. Skorobogatiy, O. Weisberg, T. D. Engeness, M. Soljacić, S. A. Jacobs, J. D. Joannopoulos, and Y. Fink, "Lowloss asymptotically single-mode propagation in large-core OmniGuide fibers," Opt. Express, vol. 9, no. 13, pp. 748-779, Dec. 2001.

[24] S. Esposito, "Universal photonic tunneling time," Phys. Rev. E, Stat. Phys. Plasmas Fluids Relat. Interdiscip. Top., vol. 64, no. 026609, 2001.

[25] J. S. Aitchison, D. C. Hutchings, J. U. Kang, G. I. Stegeman, and A. Villeneuve, "The nonlinear optical properties of AlGaAs at the half band gap," IEEE J. Quantum Electron., vol. 33, no. 3, pp. 341-348, Mar. 1997.

[26] S. Adachi, "GaAs, $\mathrm{AlAs}$, and $\mathrm{Al}_{x} \mathrm{Ga}_{1-x}$ As material parameters for use in research and device applications," J. Appl. Phys., vol. 58, no. 3, pp. R1R29, Aug. 1985

[27] J. Chilwell and I. Hodgkinson, "Thin-films field-transfer matrix theory of planar multilayer waveguides and reflection from prism-loaded waveguides," J. Opt. Soc. Amer. A, Opt. Image Sci., vol. 1, no. 7, pp. 742-753, Jul. 1984.

[28] J. Khurgin, "Improvement of frequency-conversion efficiency in waveguides with rotationally twinned layers," Opt. Lett., vol. 13, no. 7, pp. 603605, Jul. 1988

[29] T. Suhara and M. Fujimura, Waveguide Nonlinear-Optic Devices. Berlin, Germany: Springer, 2003.

[30] C. N. Ironside, J. S. Aitchison, and J. M. Arnold, "An all-optical switch employing the cascaded second-order nonlinear effect," IEEE J. Quantum Electron., vol. 29, no. 10, pp. 2650-2654, Oct. 1993.

[31] M. M. Fejer, G. A. Magel, D. H. Jundt, and R. L. Byer, "Quasi-phasematched second harmonic generation: Tuning and tolerances," IEEE J Quantum Electron., vol. 28, no. 11, pp. 2631-2654, Nov. 1992.

[32] A. Argyros, "Guided modes and loss in Bragg fibres," Opt. Express, vol. 10, no. 24, pp. 1411-1417, Dec. 2002.

[33] M. Born and E. Wolf, Principles of Optics: Electromagnetic Theory of Propagation, Interference, and Diffraction of Light, 6th ed. New York: Pergamon, 1980

[34] J.-P. Weber, "Optimization of the carrier-induced effective index change in InGaAsP waveguides - application to tunable Bragg filters," IEEE J. Quantum Electron., vol. 30, no. 8, pp. 1801-1816, Aug. 1994.

[35] G. M. Smith, J. S. Hughes, R. M. Lammert, M. L. Osowski, and J. J. Coleman, "Wavelength tunable 2-pad ridge-wave-guide distributed-Braggreflector InGaAs-GaAs quantum-well lasers," Electron. Lett., vol. 30 , no. 16, pp. 1313-1314, Aug. 1994.
[36] J. G. Mendoza-Alvarez, F. D. Nunes, and N. B. Patel, "Refractive index dependence on free carriers for GaAs," J. Appl. Phys., vol. 51, no. 8, pp. 4365-4367, Aug. 1980.

[37] H. C. Huang and S. Yee, "Change in refractive index for $p$-type GaAs at $\lambda=1.06,1.3$, and $1.55 \mu \mathrm{m}$ due to free carriers," J. Appl. Phys., vol. 70, no. 2, pp. 925-929, Jul. 1991.

[38] B. R. Bennett, R. A. Soref, and J. A. Del Alamo, "Carrier-induced change in refractive index of InP, GaAs, and InGaAsP," IEEE J. Quantum Electron., vol. 26, no. 1, pp. 113-122, Jan. 1990.

[39] N. Peyghambarian, S. W. Koch, and A. Mysyrowicz, Introduction to Semiconductor Optics. New York: Prentice-Hall, 1993.

[40] T. H. Wood, R. W. Tkach, and A. R. Chraplyvy, "Observation of large quadratic electro-optic effect in GaAs-AlGaAs multiple quantum wells," Appl. Phys. Lett., vol. 50, no. 13, pp. 798-800, Mar. 1987.

[41] C.-A. Berseth, C. Wuethrich, and F. K. Reinhart, "The electro-optic coefficients of GaAs: Measurements at 1.32 and $1.52 \mu \mathrm{m}$ and study of their dispersion between 0.9 and $10 \mu \mathrm{m}$," J. Appl. Phys., vol. 71, no. 6, pp. 2821-2825, Mar. 1992.

[42] J.-M. Yang and C.-Y. Kao, "An evolutionary algorithm for the synthesis of multilayer coatings at oblique light incidence," J. Lightw. Technol., vol. 19, no. 4, pp. 559-570, Apr. 2001.

[43] S. Martin, J. Rivory, and M. Schoenauer, "Synthesis of optical multilayer systems using genetic algorithms," Appl. Opt., vol. 34, no. 13, pp. $2247-$ 2254, May 1995.

Brian R. West received the B.Eng. degree in engineering physics from McMaster University, Hamilton, ON, Canada, in 1997, the M.S. degree from the Thayer School of Engineering, Dartmouth College, Hanover, NH, in 1999, and the M.S. and Ph.D. degrees from the Optical Sciences Center, University of Arizona, Tucson, in 2002 and 2004, respectively, where his research included modeling the processing of ion-exchanged glass waveguides and devices.

He was a Research Scientist with CoreTek, Inc., Burlington, MA, and MPB Communications, Pointe-Claire, QC, Canada, working on fiber-based devices. $\mathrm{He}$ is currently a Postdoctoral Research Fellow in the Department of Electrical and Computer Engineering, University of Toronto, Toronto, ON, Canada.

Dr. West is a Member of OSA and SPIE.

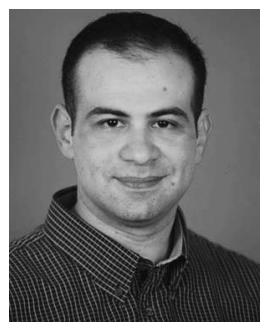

A. S. Helmy (M'99-SM'06) received the B.Sc. degree in electronics and telecommunications engineering from Cairo University, Cairo, Egypt, in 1993, and the M.Sc. and Ph.D. degrees with a focus on photonic fabrication technologies from the University of Glasgow, Glasgow, U.K., in 1994 and 1999, respectively.

Prior to his academic career, he held a position at Agilent Technologies Photonic Devices, R\&D Division, in the U.K. At Agilent, his responsibilities included developing distributed feedback lasers, monolithically integrated lasers, modulators, and amplifiers in InP-based semiconductors. He also developed high-powered submarine-class 980-nm InGaAs pump lasers. He is an Assistant Professor in the Department of Electrical and Computer Engineering, University of Toronto, Toronto, ON, Canada. His research interests include photonic device physics and characterization techniques, with emphasis on nonlinear optics in III-V semiconductors; applied optical spectroscopy in III-V optoelectronic devices and materials; and III-V fabrication and monolithic integration techniques.

Dr. Helmy is a member of the Optical Society of America. 